\title{
Low-Cost Adjustable Mandibular Advancement Device for Obstructive Sleep Apnea Patients
}

\author{
Snehal Upadhyay, MDS ${ }^{1}$; Saumyendra V. Singh, MDS²; Deeksha Arya, MDS²; Pooran Chand, MDS²; Raghuwar D. Singh, MDS² \\ ${ }^{1}$ Faculty of Dental Sciences, Dharmsinh Desai University, Nadiad, India; ${ }^{2}$ Department of Prosthodontics, King George's Medical University, \\ Lucknow, India
}

\begin{abstract}
Objectives: Nasal continuous positive airway pressure (nCPAP), though an effective therapy for obstructive sleep apnea syndrome, has a low compliance rate and is costly. As a result, newer designs of mandibular advancement devices (MADs) have gained considerable interest and are available as adjustable/nonadjustable/stock/custom-made types.

Methods: This article presents a novel economical custom-made MAD. It has an expansion screw for advancement of the mandible that has a calibration accuracy of $0.2 \mathrm{~mm}$.

Conclusions: This device is cost effective, adjustable and less bulky for obstructive sleep apnea patients.

Keywords: Adjustable mandibular advancement device, Expansion screw, Interocclusal record, Obstructive sleep apnea syndrome, Titration.

Citation: Upadhyay S, Singh, SV, Arya, D, Chand, P, Singh, RD. Low-cost adjustable mandibular advancement device for obstructive sleep apnea. J Dent Sleep Med. 2018;5(3):69-74.
\end{abstract}

Patent has been applied for on this device and the application no is IP 201611034118.

\section{INTRODUCTION}

The most effective therapeutic option for moderate obstructive sleep apnea is nasal continuous positive airway pressure (nCPAP). ${ }^{1}$ Unfortunately, people find it difficult to tolerate and compliance is low. ${ }^{2-4}$ Surgical options are effective but invasive, with associated morbidity. ${ }^{5}$

Therefore, there is considerable interest in mandibular advancement devices (MAD), which have shown significant reduction in mild to moderate sleep apnea severity. MADs dilate or open the airway by anterior positioning of the mandible during sleep to relieve snoring and OSA. ${ }^{6}$ They are especially helpful to patients intolerant to CPAP because of nasal stuffiness, dryness, and mask-related complications. ${ }^{2}$ This therapy is reportedly a suitable alternative to CPAP in patients with mild to moderate OSA because it is well tolerated by most patients. ${ }^{7}$ The device described here is a custom-made adjustable device.

This device provides adjustable levels of jaw advancement, which improves the effectiveness and comfort level of treatment because the jaw is moved only as far as required to alleviate the condition. ${ }^{8}$

\section{METHODS}

This adjustable custom-made device for dentate/ partially dentate patients consists of maxillary and mandibular acrylic plates with clasps for retention made of orthodontic wire ( $0.7 \mathrm{~mm}$ diameter), joined by two expansion screws (medium-dentaurum ref no.600-301-10) used in arch expansion appliances. The device consists of an adjustable acrylic bite block with an expansion screw embedded posterior to the corner of the mouth.

Maximum protrusion of the mandible normally ranges from 10 to $12 \mathrm{~mm}$ and MADs are normally made at $50 \%$ to $80 \%$ of this size. With the help of screws, this appliance can have a calibration accuracy of up to 0.2 $\mathrm{mm}$. It has a simple design and is relatively easy to fabricate in any dental setup. The steps to fabricate the appliance are as follows:

1. Alginate impressions (DPI Algitex) of maxillary and mandibular arches were made and poured.

2. An interocclusal record was made at $50 \%$ of maximal mandibular protrusion and $20 \%$ of maximum interincisal opening 9 (Figure 1). Twenty percent of the maximum interincisal opening is taken so as to just accommodate the expansion screw assembly. The necessary interincisal opening was calculated after measuring the maximal opening distance between the mesioincisal edge of the upper right central incisor and the mesioincisal edge of the lower right central incisor, using a Boley gauge (Neiko, USA). ${ }^{10}$ The Boley gauge was used to calculate necessary protrusive distance after measuring the distance moved by the mandibular right central incisor from centric occlusion to maximum protrusion. Casts were 
mounted on a free-plane articulator using the interocclusal record. ${ }^{11}$

3. Adam's and pinhead clasps were fabricated on posterior teeth on both sides in both arches for retention.

4. Maxillary and mandibular base plates were waxed on the casts, processed, finished, and polished separately in clear heat polymerized acrylic resin (Trevalon, Dentsply). (Figures 2 and 3)

5. Acrylic blocks to be attached to maxillary and mandibular plates were fabricated. The blocks were waxed on either side of the key slot end of an expansion screw (the dimension of each block was just enough to contain the screw and attach it to the maxillary or mandibular base plate) and impression of the waxup was taken in polyvinyl siloxane impression material. By this, a mold was fabricated for making acrylic blocks.

6. Acrylic blocks are placed around the expansion screw (medium-dentaurum ref no.600-301-10). Two such molds (one for either side of the mouth) were made (Figure 4).

7. In this device width, length, and thickness of the expansion screw is $7.3 \mathrm{~mm}, 11 \mathrm{~mm}$, and $3.1 \mathrm{~mm}$, respectively.

8. The screw was placed into the mold and autopolymerizing resin (Trevalon, Dentsply) was poured into the mold.

9. After polymerization, the acrylic blocks with the screw were removed from the mold and finished.

10. The screw block assembly was attached on both sides to the maxillary and mandibular plates at the premolar region with autopolymerizing resin (Trevalon, Dentsply) on the articulator at the recorded mandibular protrusion and opening (Figure 5).

11. Care was taken to attach the acrylic expansion screw so that the arrow on the expansion screw was facing outward (so as to be easily seen).

12. The device was finished and polished. When the key provided with the screw is moved in the direction of the arrow, the mandibular component will move into a more forward position ( $0.8 \mathrm{~mm}$ per turn of the key). Thus, the appliance can be easily titrated as required (Figure 6).

\section{DISCUSSION}

1. This device for treatment of dentate/ partially dentate OSA patients incorporates an expansion screw as a method of adjustment.

2. The device can have a calibration accuracy of up to $0.2 \mathrm{~mm}$ (one-fourth turn of the key). Therefore, the amount of advancement can be calibrated very accurately as per device efficacy and patient comfort.

3. MADs are classified as stock and custom made. The customized appliance is quite inexpensive (INR 500 or less than $\$ 9.00$ per appliance) compared to other appliances; the dorsal appliance, for instance, costs approximately \$349.00 (Dynaflex; Dynaflex Manufacturing, St Ann, MO). ${ }^{12}$

4. The advancement device described by Rico and Patel (MAP Laboratory, Buford, GA) has a ball-like projection from an acrylic plate with elastics to retain the device. This device uses tooth surface coverage for retention. ${ }^{13}$ Our design would have less tooth coverage because orthodontic wire is used for retention of the prosthesis. This appliance should be more comfortable than that described by Lamberg (Space Maintainers Laboratory, Chatsworth, CA) because it provides tongue space. ${ }^{14}$

5. This device has an advantage over designs that have the advancement screw placed in the anterior region protruding extraorally, leading to limited room for the tongue, lips, and gums [Thornton Adjustable Positioner (TAP) (Roswell Dental Care, Roswell, GA)] ${ }^{15}$ Such designs can be irritating and uncomfortable for patients.

6. The adjustable dorsal appliance ${ }^{16}$ (Space Maintainers Laboratory, Chatsworth, CA) also uses an expansion screw for adjustment. However, it is a twopiece appliance with dorsal fins as a means of engaging the maxillary and mandibular components. This requires dexterity on the part of the patient for intraoral assembly, compared to a one-piece appliance. Further, the dorsal fins project up and out from the mandibular plate, which may irritate or create trauma upon placement/removal. Last, the dual-laminate surfaces of the appliance (inner resilient and outer acrylic) make it costlier than our design.

\section{CONCLUSION}

A technique to fabricate a cost-effective, finely calibratable custom-made MAD, which is easy to adjust, has been described. 


\title{
REFERENCES
}

1. Gordon P, Sanders MH. Sleep 7: Positive airway pressure therapy for obstructive sleep apnoea/hypopnoea syndrome. Thorax 2005;60(1):68-75.

2. Kalan A, Kenyon GS, Seemungal TA, Wedzicha JA. Adverse effects of nasal continuous positive airway pressure therapy in sleep apnoea syndrome. J Laryngol Otol. 1999;113(10):888-892.

3. Yetkin O., Kunter E, Gunen H. CPAP compliance in patients with obstructive sleep apnea syndrome. Sleep Breath. 2008,12(4):365-367.

4. Bachour A1, Vitikainen P, Virkkula P, Maasilta P. CPAP interface: satisfaction and side effects. Sleep Breath. 2013 ;17(2):667-672.

5. Bonham PE, Currier GF, Orr WC, Othman J, Nanda RS. The effect of a modified functional appliance on obstructive sleep apnea. Am $J$ Orthod Dentofac Orthop. 1988;94(5):384-392.

6. Marklund M, Franklin KA, Sahlin C, Lundgren R. The effect of a mandibular advancement device on apneas and sleep in patients with obstructive sleep apnea. Chest. 1998;113(3):707-713.

7. Tan YK, L'Estrange PR, Luo YM, et al. Mandibular advancement splints and continuous positive airway pressure in patients with obstructive sleep apnoea: a randomized cross-over trial. Eur J Orthod. 2002;24(3):239-249,

8. Kyung SH, Park Y, Pae E. Obstructive sleep apnea patients with the oral appliance experience pharyngeal size and shape changes in three dimensions. Angle Orthod. 2005;75(1):15-22.

9. Rashmikant US, Chand P, Singh SV, Singh RD, Arya D, Kant S,
Agarwal SP. Cephalometric evaluation of mandibular advancement at different horizontal jaw positions in obstructive sleep apnoea patients: a pilot study. Aust Dent J. 2013;58(3):293-300.

10. Li Y, Jia C, Zhang Z. The normal range of maximum mouth opening and its correlation with height or weight in the young adult Chinese population. J Dent Sci. 2017;12:56-59.

11. Pitsis AJ, Darendeliler MA, Gotsopoulos H, Petocz P, Cistulli PA. Effect of vertical dimension on efficacy of oral appliance therapy in obstructive sleep apnea. Am $J$ Respir Crit Care Med. 2002;166(6):860-864.

12. Johal A, Haria P, Manek S, Joury E, Riha R. Ready-made versus custom-made mandibular repositioning devices in sleep apnea: a randomized clinical trial. J Clin Sleep Med. 2017;15;13(2):175-182.

13. Rico H, Patel $M$. Intraoral apparatus for managing snoring and obstructive sleep apnea. US20150272773A1. 2015-10-01.

14. Lamberg S. Intraoral mandibular advancement device for treatment of sleep disorders, including snoring, obstructive sleep apnea, and gastroesophageal reflux disease and method for delivering the same. US20070079833A1.2007-04-12.

15. Lettieri CJ, Paolino N, Eliasson AH, Shah A, Holley AB Comparison of adjustable and fixed oral appliances for the treatment of obstructive sleep apnea. J Clin Sleep Med. 2011;7(5):439-445.

16. Quaka GW, Buddemeyer DE. Anti-snoring and sleep apnea device. US20140326252A1. 2014-11-06

\section{SUBMISSION AND CORRESPONDENCE INFORMATION}

\author{
Submitted for publication January 9, 2018 \\ Submitted in final revised form May 9, 2018 \\ Accepted for publication June 26, 2018
}

Address correspondence to: Deeksha Arya, MDS; A-401, Indraprastha Estate, Faizabad Road, Lucknow 226007, Email: deeksha.arya@gmail.com

\section{DISCLOSURE STATEMENT}

The authors have no financial conflicts of interest to disclose. 


\section{APPENDIX}

Images of Devices

Figure 1 - Interocclusal Record at $50 \%$ of Mandibular Protrusion and $20 \%$ of Interincisal opening

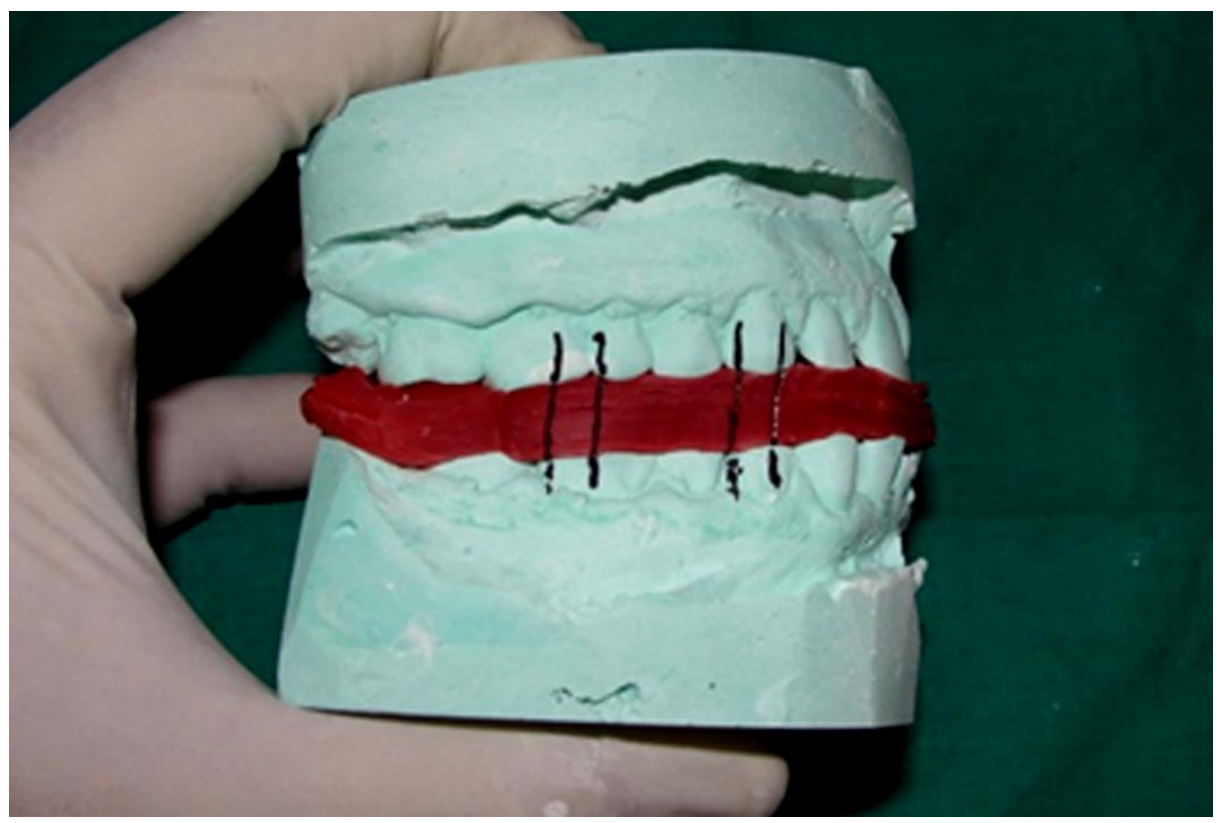

Figure 2 - Acrylic Plates with Wire Elements

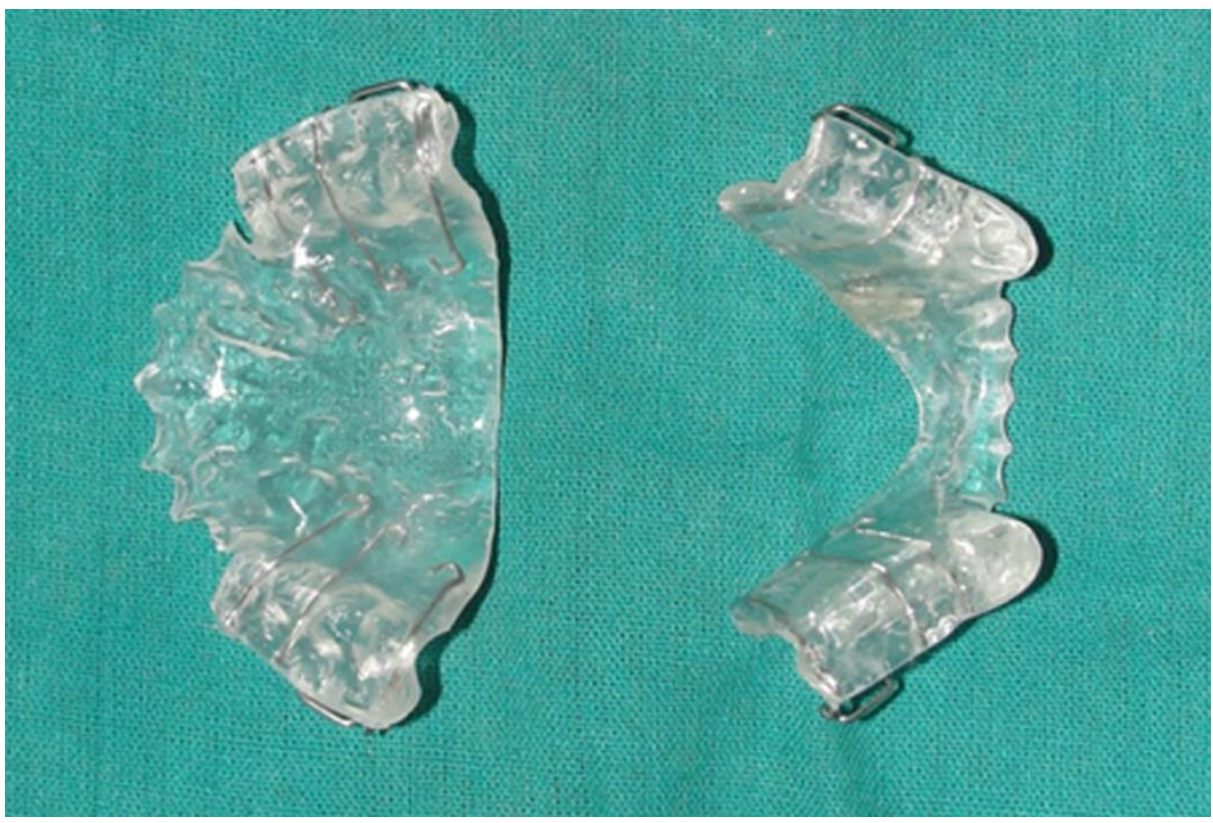


Figure 3 - Acrylic Plates with Wire Elements

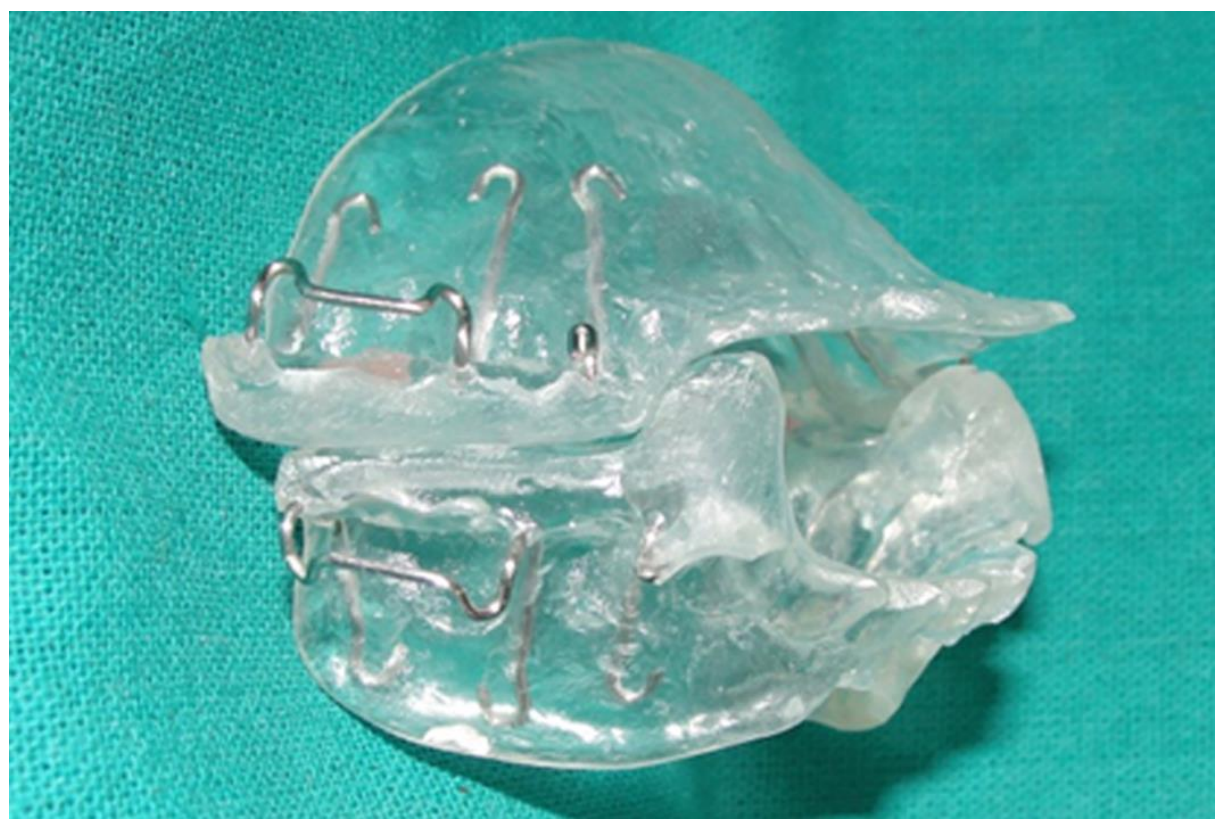

Figure 4 - Expansion Screw with Key and Silicone Mold

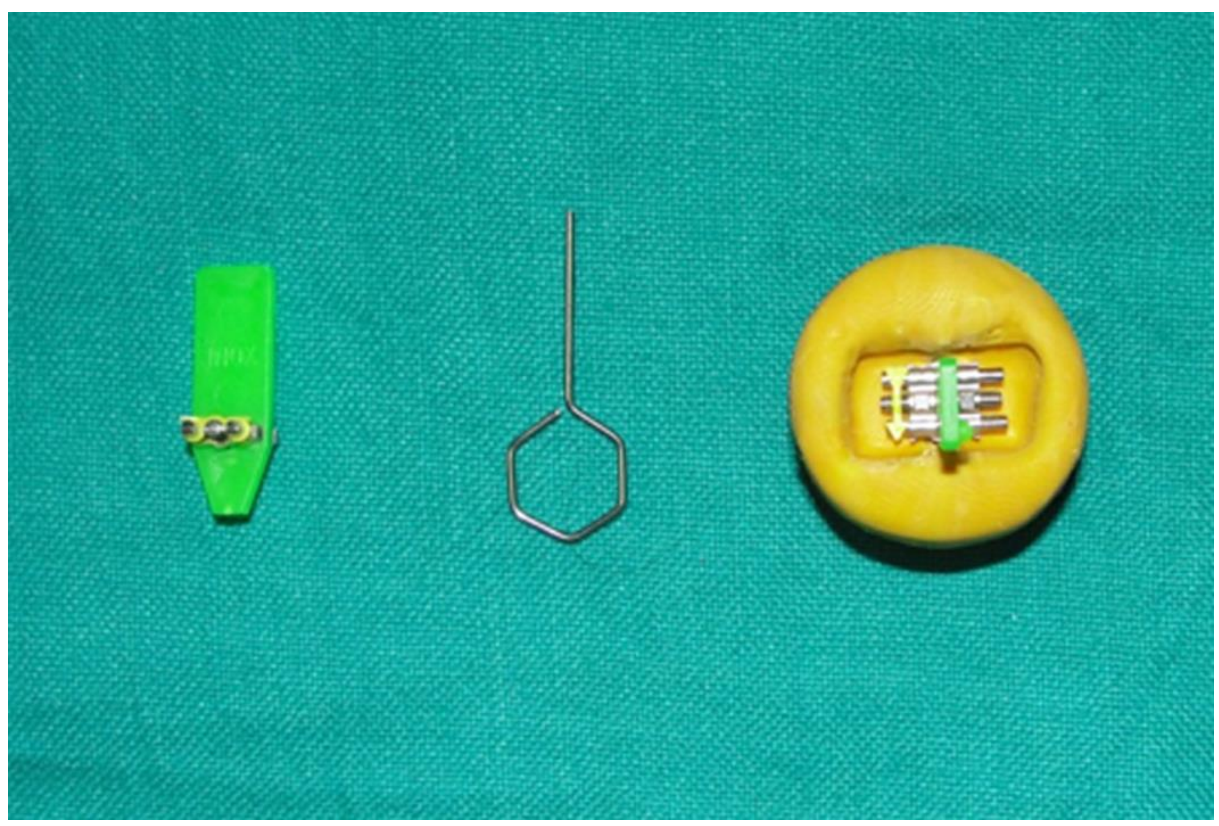


Figure 5 - Appliance Positioned in Mouth

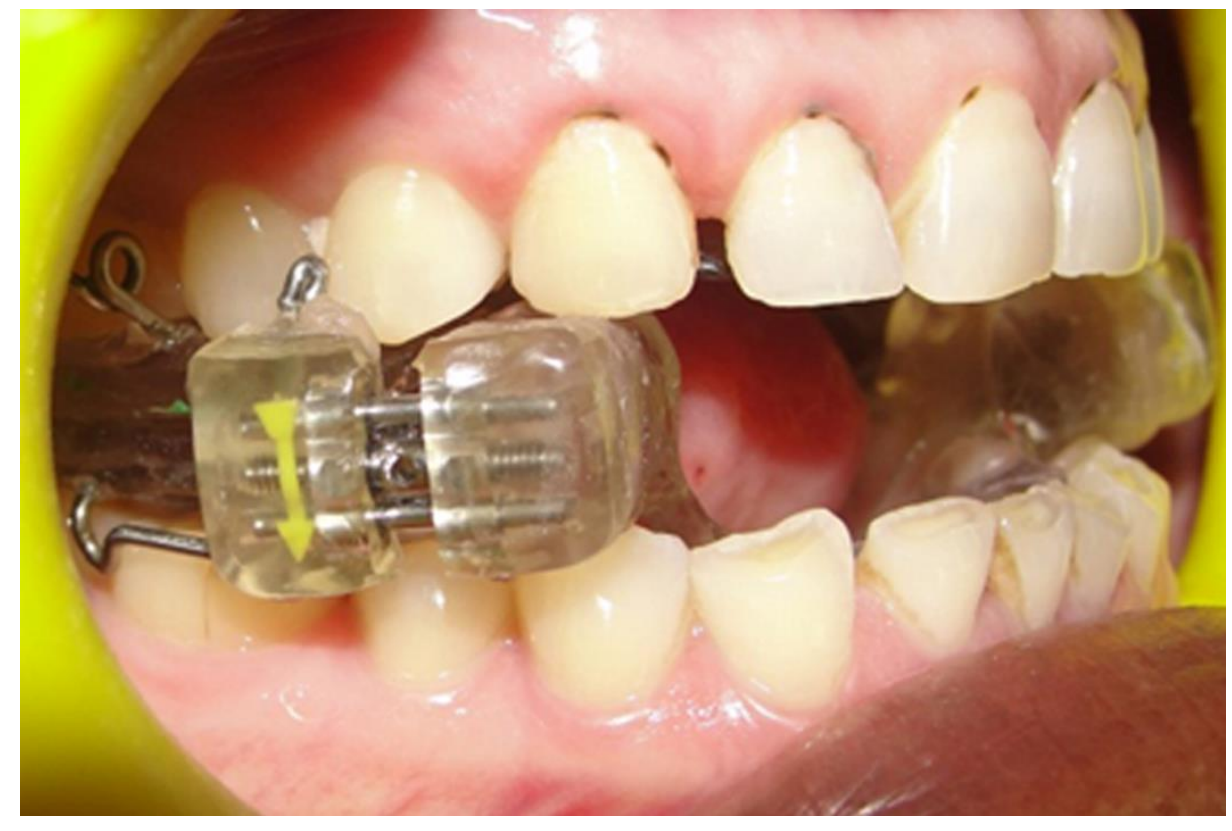

Figure 6 - Direction of Movement of Appliance

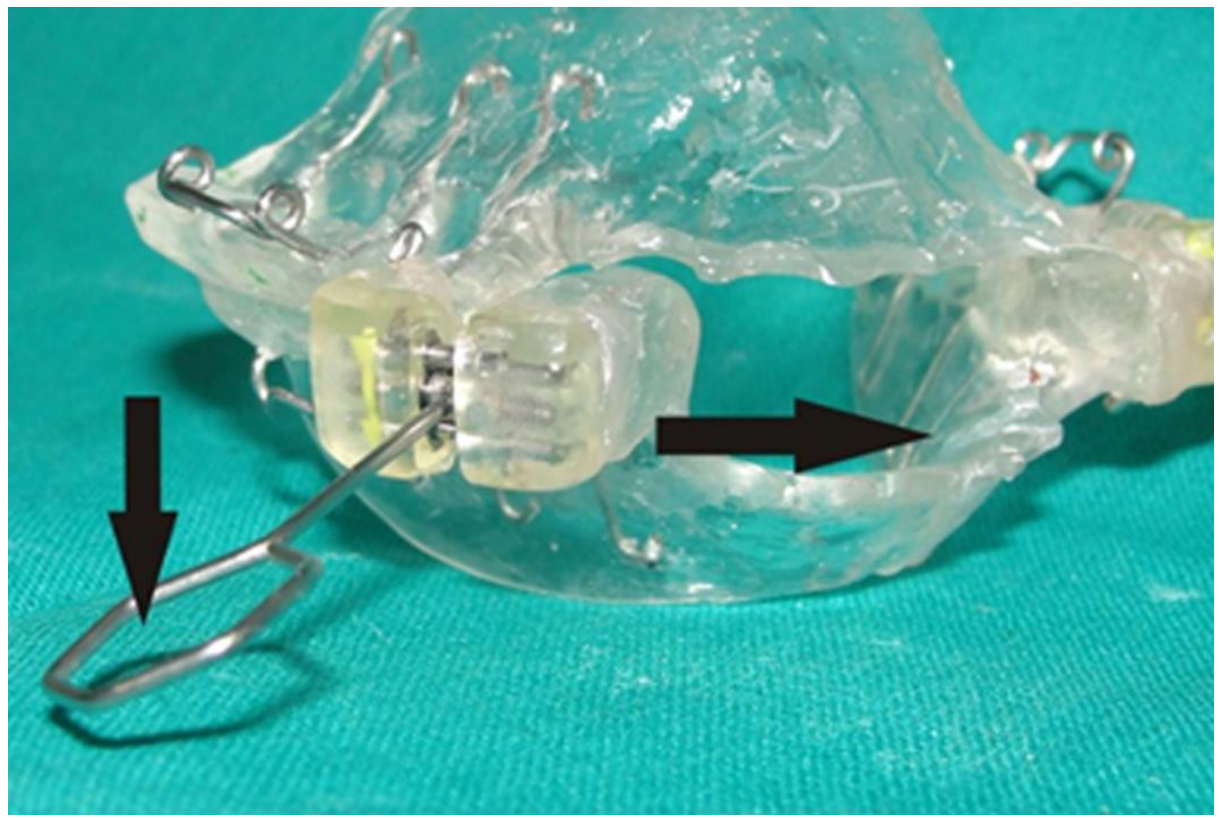

